\title{
The plastic liver: differentiated cells, stem cells, every cell?
}

\author{
Christopher J. Hindley, ${ }^{1,2,3,4}$ Gianmarco Mastrogiovanni, ${ }^{1,2}$ and Meritxell Huch ${ }^{1,2,3}$ \\ ${ }^{1}$ Wellcome Trust/Cancer Research UK Gurdon Institute, ${ }^{2}$ Wellcome Trust/MRC Stem Cell Institute, and ${ }^{3}$ Department of Physiology, Development and Neuroscience, University of Cambridge, \\ Cambridge, United Kingdom. ${ }^{4}$ Cavendish Laboratory, Department of Physics, Cambridge, United Kingdom.
}

\begin{abstract}
The liver is capable of full regeneration following several types and rounds of injury, ranging from hepatectomy to toxin-mediated damage. The source of this regenerative capacity has long been a hotly debated topic. The damage response that occurs when hepatocyte proliferation is impaired is thought to be mediated by oval/dedifferentiated progenitor cells, which replenish the hepatocyte and ductal compartments of the liver. Recently, reports have questioned whether these oval/progenitor cells truly serve as the facultative stem cell of the liver following toxin-mediated damage. In this issue of the $\mathrm{JCl}$, Kordes and colleagues use lineage tracing to follow transplanted rat hepatic stellate cells, a resident liver mesenchymal cell population, in hosts that have suffered liver damage. Transplanted stellate cells repopulated the damaged rat liver by contributing to the oval cell response. These data establish yet another cell type of mesenchymal origin as the progenitor for the oval/ductular response in the rat. The lack of uniformity between different damage models, the extent of the injury to the liver parenchyma, and potential species-specific differences might be at the core of the discrepancy between different studies. Taken together, these data imply a considerable degree of plasticity in the liver, whereby several cell types can contribute to regeneration.
\end{abstract}

\section{A novel player in an old game?}

Approximately $80 \%$ of liver mass is composed of hepatocytes, which are responsible for the majority of liver-associated functions, including metabolic and detoxification activities. The remainder of the tissue is composed of biliary epithelial cells (BECs) (bile ducts), blood vessels, Kupffer cells, and hepatic stellate cells (HSCs). The liver possesses a remarkable regenerative capacity and can fully recover from multiple rounds of tissue removal (up to $70 \%$ ). It is accepted and demonstrated for all vertebrates studied that hepatocyte hypertrophy and proliferation are responsible for the liver regeneration that preserves the hepato- cyte compartment following partial hepatectomy or toxic damage (Figure 1A). However, during toxin-mediated damage, which impairs hepatocyte proliferation, it is not completely clear how the liver regenerates, and the field has been divided on this issue. The controversy arises from questions in two distinct areas. First, is a facultative liver stem/ progenitor cell required to repopulate the liver? Second, what is the cell type that originates such a stem/progenitor cell population? Recent studies in murine models, a system amenable to multiple Cre-based lineage-tracing approaches, show that hepatocytes alone contribute to tissue regeneration, even following toxin-

\section{Related Article: p. 5503}

Authorship note: Christopher J. Hindley and Gianmarco Mastrogiovanni contributed equally to this work.

mediated damage $(1,2)$. These studies are in contrast to previous reports that demonstrated BECs or progenitor cells regenerating the tissue after damage, albeit in minor fractions $(2 \%-3.2 \%$; refs. $3-5$, and reviewed in ref. 6). Interestingly, another recent report indicates that ablation of hepatic progenitor cells and their descendants impairs liver regeneration (7), again questioning the claim that a ductular reaction is not required for liver regeneration $(1,2)$.

Initial historic studies in rats noted the appearance of a transient, rapidly proliferating cell type that expresses both BEC- and hepatocyte-specific markers as well as the embryonic liver marker $\alpha$-fetoprotein. These cells were named oval cells or reactive ductal cells, mainly because their appearance is associated with the regenerative process termed the "ductular reaction" $(6,8) .\left[{ }^{3} \mathrm{H}\right]$-thymidine-labeling studies provided evidence that this transit-amplifying cell population repopulates the rat liver by generating novel hepatocytes $(9,10)$. These results are consistent with data obtained in the zebrafish, in which complete depletion of the hepatocyte compartment results in ductal cells activating a progenitor program, resulting in restoration of the lost hepatocytes. Further, ductular reactions have also been identified in virtually all human liver disorders that involve cell loss such as fulminant liver failure (11), suggesting that the process is linked to regeneration of the liver (Figure 1). In this issue, Kordes and colleagues enter this controversial arena and identify the mesenchymal HSC as a potential source of cells for the regenerating rat liver (12).

\section{HSCs: a mesenchymal} progenitor source for the liver HSCs are a liver-resident mesenchymal stem cell (MSC) population that expresses markers of multiple germ layers. Chronic activation of HSCs promotes differenti- 
A
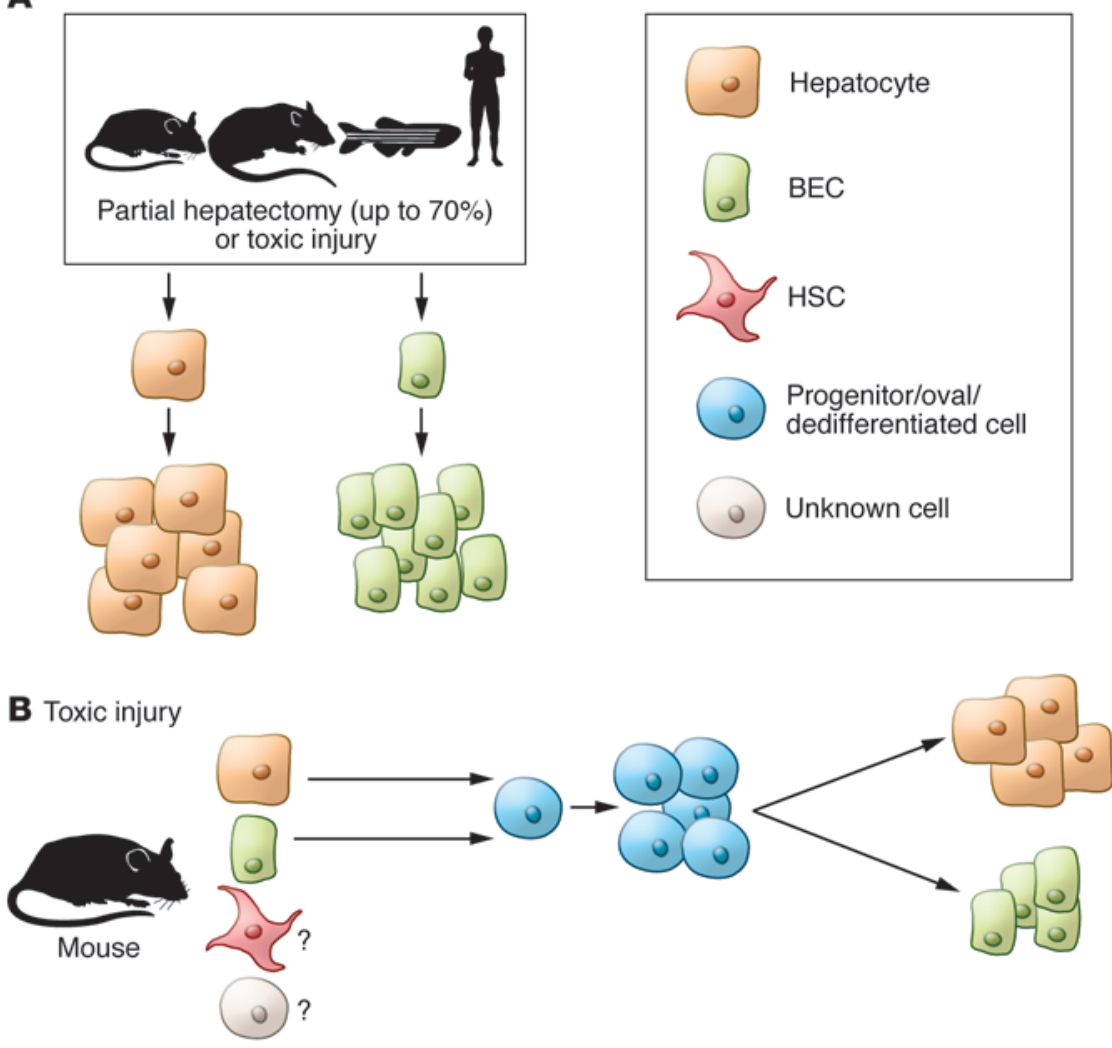

C Toxic injury (impaired hepatocyte proliferation)

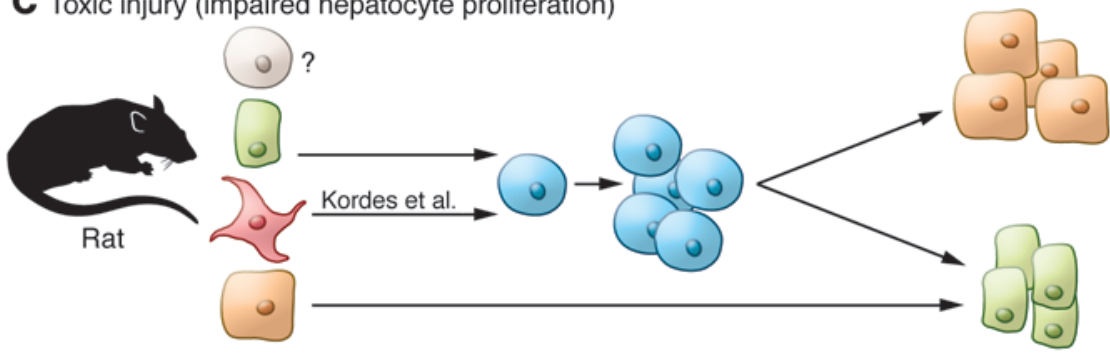

D Toxic injury (95\% hepatocyte loss)

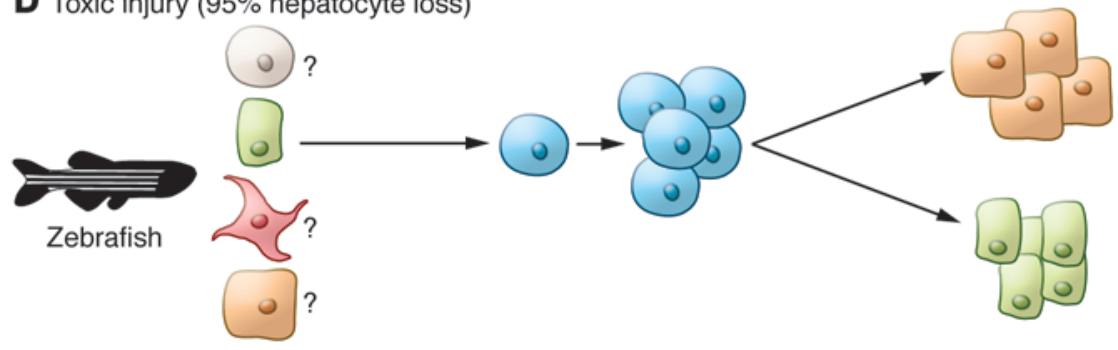

E Fulminant liver failure (up to $80 \%$ hepatocyte loss)

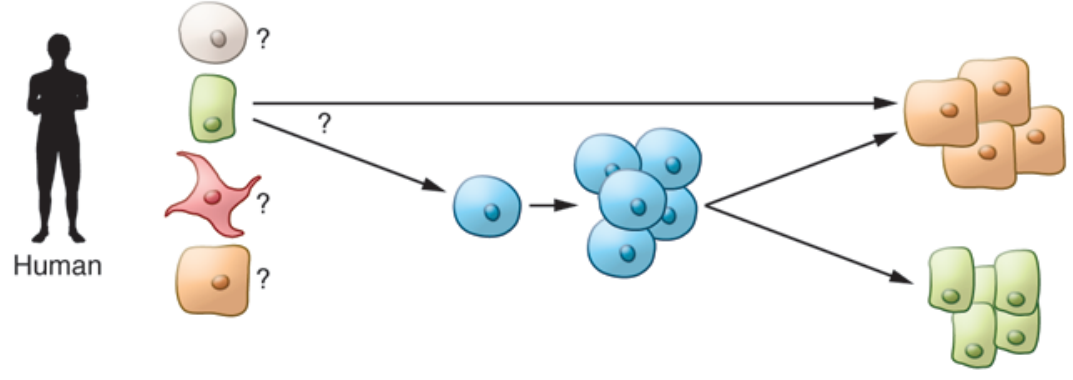

Figure 1. Schematic representation of cell regeneration in the liver after damage. (A) Partial hepatectomy or toxic injury activates hepatocytes and BECs, which are able to regenerate by self-duplication in all vertebrates studied. (B) Liver regeneration in mouse models after toxic injury. Lineage tracing in mice has demonstrated a contribution of both hepatocytes and $B E C s$ (at low frequencies) to the generation of progenitor and oval cells after damage (reviewed in ref. 6). (C) The work of Kordes et al. (12) suggests a role for HSCs in the repair process after toxic injury in the rat, joining BECs as a potential source of progenitor cells. Transdifferentiation of hepatocytes into BECs has been observed in the rat after bile duct ligation coupled with biliary toxin methylene diamiline (DAPM) pretreatment (27). (D) Activation of BECs to a progenitor state has been observed after toxic injury and $95 \%$ hepatocyte loss in the zebrafish liver (20). (E) The human liver can recover from fulminant liver failure that can cause up to $80 \%$ hepatocyte loss. A strong ductular reaction is observed, with $30 \%$ of the cells expressing markers of BEC and hepatocyte lineages. BECs seem to be involved in the regeneration process; however, it cannot be formally proved whether $B E C s$ generate hepatocytes. Further studies are needed to understand whether this process occurs through direct transdifferentiation or generation of a progenitor cell (11). 
ation into myofibroblasts, which induce fibrogenesis in the injured liver $(13,14)$. Recent reports have both supported (15) and refuted $(1,16)$ a role for the mesenchymal HSC population as a facultative liver stem cell. In order to address the contribution of HSCs to liver regeneration, Kordes and colleagues transplanted HSCs into rats with liver injury and determined that the transplanted HSCs were a source of both hepatocytes and BECs in the regenerating liver (12). Specifically, the authors transplanted $\mathrm{GFP}^{+}$HSCs from male rats into female GUNN rat recipients, allowing both GFP expression and the Y chromosome to be used as tracing tools. HSCs were seen to contribute to hepatocytes and bile ducts following partial hepatectomy and administration of 2-acetylaminofluorene or retrorsine, both of which are injury models that prevent hepatocyte proliferation and favor ductular reaction (17). Hepatocyte maturity was assessed by rescue of the bilirubin conjugation defect in the GUNN-mutant rat recipients and by the release of albumin and bile acids following in vitro differentiation of HSCs into hepatocytes. In addition, the capacity of HSCs to be retransplanted was tested by taking $\mathrm{GFP}^{+}$donor cells from host $\mathrm{BM}$ and retransplanting them into a second recipient, in which the $\mathrm{GFP}^{+}$cells were determined to contribute to liver regeneration. Therefore, the study by Kordes et al. indicates that HSCs act as a liver stem cell pool and are capable of contributing to repeated rounds of regeneration (12).

Although cell fusion cannot be completely ruled out as a mechanism of liver regeneration following transplantation, since Kordes and colleagues did not perform female-to-male transplantation, their in vitro data argue that HSCs undergo a mesenchymal-to-epithelial transition (MET) during their differentiation into $\mathrm{EpCAM}^{+}$ cells, as evidenced by expression of ductal cell and hepatocyte markers. In addition, using super-resolution microscopy, Kordes and colleagues noted the presence of $\mathrm{GFP}^{+}$ cells with epithelial characteristics during ductular reaction and that the HSC marker desmin was present in scattered keratin-19+ oval cells undergoing ductular reactions. In this model, the activated HSCs would downregulate mesenchymal markers and upregulate biliary epithelial markers, such as keratin-19, before contributing to ductular reactions as part of the oval cell pop- ulation. Thus, Kordes et al. elegantly connect the seemingly contradictory previous reports, which either supported or refuted a role of HSCs in liver regeneration (12).

Despite the apparent demonstration that HSCs contribute to liver regeneration, several questions remain. Since HSCs are resident in the liver, do they participate in homeostatic turnover? And upon liver damage, do HSCs contribute to regeneration or do they do so only when transplanted? Given the systemic nature of the transplantation used in this report, it is reasonable to question the relative contribution of each of these cell populations to the regenerating liver. Further studies will be required to elucidate in vivo, in rat models of impaired hepatocyte proliferation, whether resident HSCs also contribute to regeneration of the tissue. In this regard, it is interesting to note that the isolated MSCs used by Kordes and colleagues for in vitro differentiation had the capacity to undergo MET and differentiate into functional hepatocytes. If the ability to undergo MET is a general property of MSCs, then we again return to the question of the relative contribution of resident HSCs and other MSC pools to liver regeneration.

Interestingly, Kordes et al. observed that the proportion of cells engrafted following transplantation was dependent on the injury model used, suggesting that different progenitor sources drive regeneration in response to various types of injury, a theory consistent with other reports $(7,18)$. If this is the case, it will be important to define the signals that activate each progenitor type in response to a specific injury. A better knowledge of the signals that activate different progenitor cell populations may be useful for the treatment of chronic liver injuries, in which the long-term activation of HSCs is known to promote fibrogenesis and downregulation of HSC activity is desirable (14).

\section{Liver plasticity, a unifying theory?}

The lack of uniformity between different damage models, the extent of the damage to the liver parenchyma, and potential species-specific differences lie at the heart of the controversy. It is noteworthy that data from several species, including rodents (reviewed in ref. 19), zebrafish (20), and humans (11), are all consistent with a contribution to regeneration from ductular reactions; however, questions remain about the cell source and how much a given cell type contributes to regeneration rather than about the regeneration process as a whole.

One way to reconcile the data provided by Kordes and colleagues with other reports could be to view the liver as a highly plastic organ. If we consider that the damaged liver has plasticity with regard to the cell of origin for regeneration, then depending on the extent and type of damage, several cell types could act as progenitor populations to mediate ductular reactions; therefore, a different interpretation of the many conflicting reports becomes possible. A process that is capable of drawing on several cell populations for organ regeneration is not without precedent. In the intestine, the crypt basal columnar (CBC) cell is the cell type responsible for organ homeostasis. This cell population was described by Leblond (21) and confirmed as a bona fide stem cell in the gut by elegant lineage-tracing studies (22). Although the CBC cell is the workhorse of intestinal proliferation under normal conditions, ablation of the CBC cell compartment results in several other cell populations, including, but probably not limited to, secretory precursors (23), label-retaining cells (24), and +4 cells (25), activating a facultative stem cell program to maintain organ function. It would appear that this plasticity is not just a function of endoder$\mathrm{m}$-derived organs, as similar mechanisms that result in use of more than one stem cell pool exist in the skin (26).

It seems reasonable to speculate that the ability to draw upon several "back-up" cell sources to maintain organ function following damage would be a favorable evolutionary strategy. The ability of multiple cell sources to provide regenerative capacity would allow flexibility against several forms of injury, especially injuries that might ablate specific cell pools but leave others intact. Viewed in terms of facultative progenitor cell plasticity, previous reports would suggest that the liver is a highly plastic organ and able to draw upon varying cell sources for regeneration, depending on the type and extent of the damage. We can now add the study by Kordes et al. (12), which demonstrates that a mesenchymal cell source contributes 
to the regenerating liver during ductular reactions, to the growing list of studies that support plasticity in the liver.

\section{Acknowledgments}

C.J. Hindley is supported by the Herchel Smith Fund and G. Mastrogiovanni by an FP7 Marie Curie Initial Training Network grant (WntsApp). M. Huch is a Wellcome Trust Sir Henry Dale Fellow and is jointly funded by the Wellcome Trust and the Royal Society (104151/Z/14/Z). We thank Ben Simons and Bon-Kyoung Koo for their support and advice.

Address correspondence to: Meritxell Huch, Gurdon Institute, University of Cambridge, Tennis Court Road, CB21QN, United Kingdom. Phone: 01223334088; E-mail:m.huch@gurdon.cam.ac.uk.

1. Schaub JR, Malato Y, Gormond C, Willenbring $\mathrm{H}$. Evidence against a stem cell origin of new hepatocytes in a common mouse model of chronic liver injury. Cell Rep. 2014;8(4):933-939.

2. Yanger K, et al. Adult hepatocytes are generated by self-duplication rather than stem cell differentiation. Cell Stem Cell. 2014;15(3):340-349.

3. Dorrell C, et al., Prospective isolation of a bipotential clonogenic liver progenitor cell in adult mice. Genes Dev. 2011;25(11):1193-1203.

4. Huch M, et al. In vitro expansion of single Lgr5+ liver stem cells induced by Wnt-driven regeneration. Nature. 2013;494(7436):247-250.

5. Español-Suñer R, et al. Liver progenitor cells yield functional hepatocytes in response to chronic liver injury in mice. Gastroenterology. 2012;143(6):1564-1575.
6. Williams MJ, Clouston AD, Forbes SJ. Links between hepatic fibrosis, ductular reaction, and progenitor cell expansion. Gastroenterology. 2014;146(2):349-356

7. Shin S, Upadhyay N, Greenbaum LE, Kaestner $\mathrm{KH}$. Ablation of Foxl1-Cre-labeled hepatic progenitor cells and their descendants impairs recovery of mice from liver injury. Gastroenterology. 2014;pii:S0016-5085(14)01193-7.

8. Farber E. Similarities in the sequence of early histological changes induced in the liver of the rat by ethionine, 2-acetylamino-fluorene, and 3'-methyl-4-dimethylaminoazobenzene. Cancer Res. 1956;16(2):142-148.

9. Evarts RP, Nagy P, Marsden E, Thorgeirsson SS. A precursor-product relationship exists between oval cells and hepatocytes in rat liver. Carcinogenesis. 1987;8(11):1737-1740.

10. Evarts RP, Nagy P, Nakatsukasa H, Marsden E, Thorgeirsson SS. In vivo differentiation of rat liver oval cells into hepatocytes. Cancer Res. 1989;49(6):1541-1547.

11. Hattoum A, Rubin E, Orr A, Michalopoulos GK. Expression of hepatocyte epidermal growth factor receptor, FAS and glypican 3 in EpCAMpositive regenerative clusters of hepatocytes, cholangiocytes, and progenitor cells in human liver failure. Hum Pathol. 2013;44(5):743-749.

12. Kordes C, Sawitza I, Götze S, Herebian D, Häussinger D. Hepatic stellate cells contribute to progenitor cells and liver regeneration. J Clin Invest. 2014;124(12):5503-5515.

13. Friedman SL, Roll FJ, Boyles J, Bissell DM. Hepatic lipocytes: the principal collagen-producing cells of normal rat liver. Proc Natl Acad Sci U S A. 1985;82(24):8681-8685.

14. Mederacke I, et al. Fate tracing reveals hepatic stellate cells as dominant contributors to liver fibrosis independent of its aetiology. Nat Com mun. 2013;4:2823.

15. Swiderska-Syn M, et al. Myofibroblastic cells function as progenitors to regenerate murine livers after partial hepatectomy. Gut.
2014;63(8):1333-1344

16. Lua I, James D, Wang J, Wang KS, Asahina K. Mesodermal mesenchymal cells give rise to myofibroblasts, but not epithelial cells, in mouse liver injury. Hepatology. 2014;60(1):311-322.

17. Tatematsu M, Ho RH, Kaku T, Ekem JK, Farber E. Studies on the proliferation and fate of oval cells in the liver of rats treated with 2-acetylaminofluorene and partial hepatectomy. Am J Pathol. 1984;114(3):418-430.

18. Rodrigo-Torres D, et al. The biliary epithelium gives rise to liver progenitor cells. Hepatology. 2014;60(4):1367-1377.

19. Michalopoulos GK. The liver is a peculiar organ when it comes to stem cells. Am J Pathol. 2014;184(5):1263-1267.

20. Choi TY, Ninov N, Stainier DY, Shin D. Extensive conversion of hepatic biliary epithelial cells to hepatocytes after near total loss of hepatocytes in zebrafish. Gastroenterology. 2014;146(3):776-788.

21. Cheng H, Leblond CP. Origin, differentiation and renewal of the four main epithelial cell types in the mouse small intestine. I. Columnar cell. Am J Anat. 1974;141(4):461-479.

22. Barker $\mathrm{N}$, et al. Identification of stem cells in small intestine and colon by marker gene Lgr5. Nature. 2007;449(7165):1003-1007.

23. van Es JH, et al. Dll1+ secretory progenitor cells revert to stem cells upon crypt damage. Nat Cell Biol. 2012;14(10):1099-1104.

24. Buczacki SJ, et al. Intestinal label-retaining cells are secretory precursors expressing Lgr5. Nature. 2013;495(7439):65-69.

25. Tian $\mathrm{H}$, et al. A reserve stem cell population in small intestine renders Lgr5-positive cells dispensable. Nature. 2011;478(7368):255-259.

26. Blanpain C. Stem cells: Skin regeneration and repair. Nature. 2010;464(7289):686-687.

27. Michalopoulos GK, Barua L, Bowen WC. Transdifferentiation of rat hepatocytes into biliary cells after bile duct ligation and toxic biliary injury. Hepatology. 2005;41(3):535-544 